\title{
Chapter 16 \\ Rapid Screening of Chemical Sensing \\ Materials Using Molecular Modeling Tools for the JPL Electronic Nose
}

\author{
Abhijit V. Shevade, Margie L. Homer, and William A. Goddard III
}

\begin{abstract}
We report a first principles Quantum Mechanics (QM) study to screen chemical functionalities in polymer-based chemical sensing materials to detect sulfur dioxide $\left(\mathrm{SO}_{2}\right)$ and elemental mercury $(\mathrm{Hg})$ vapors. The screening methodology involves evaluating the performance of various chemical functionalities in polymers based on their binding energy scores of the target molecules $\left(\mathrm{SO}_{2}\right.$ and $\left.\mathrm{Hg}\right)$. The QM results were validated by comparing the actual sensor response trends with the calculated binding energy values, by performing experiments using polymer-carbon composite sensors made from the polymers with the recommended chemical functionalities. A good correlation is found between the experimental sensor responses (strong or weak) to $\mathrm{SO}_{2}$ and $\mathrm{Hg}$ and the calculated binding energy values (strong or weak). The sensors were successfully used in the Third Generation JPL Electronic Nose (ENose) Technology Demonstration Experiment on the International Space Station (ISS).
\end{abstract}

\subsection{Introduction}

The screening and selection of chemical sensing materials is a multi-step process. The process starts with identifying potential sensing material candidates and evaluating the materials using both chemical modeling approaches and laboratory experiments. Narrowing the sensor set to the most promising candidates is subsequently based on the experimental data and statistical evaluation of those data. The choice of the final set of materials is made after performing reliability studies and performance comparisons. Experimental techniques such as high throughput (HT) screening, which applies combinatorial strategies to screen large sets (tens and hundreds) of

\footnotetext{
A. V. Shevade $(\varangle) \cdot$ M. L. Homer

Jet Propulsion Laboratory (JPL), California Institute of Technology, 4800 Oak Grove Drive, Pasadena, CA 91109, USA

e-mail: Abhijit.Shevade@jpl.nasa.gov

W. A. Goddard III

Materials and Process Simulation Center, California Institute of Technology, Pasadena, CA 91125, USA
} 
sensing materials are very popular [1-3]. HT experiments use sensing arrays coated with various materials, which are tested at the desired process and varying operating conditions. These procedures are very elaborate and intensive. Hence, rapid initial screening to identify potential sensing material candidates is needed.

A rapid theoretical approach to screening would include evaluating the binding energies between sensing materials and target analytes. On a fundamental level, the properties of materials depend on understanding the chemical interactions, which involves understanding their electronic and atomic level descriptions. On a lengthtime scale, this would mean considering descriptions at the nanometer-picosecond level. The hierarchy of the materials simulations to obtain engineered materials of desired properties [4], starts with first principle quantum mechanics (electrons), followed by Molecular Dynamics (atoms), then Meso-scale (segments/grain), and finally Finite element analysis (grids) level description for predicting properties of new materials.

The QM calculations are the most accurate, and the time for QM simulation scales as $\mathrm{N}^{3}-\mathrm{N}^{5}$ for most the characteristic methods ( $\mathrm{N}$ system size), but new approaches have achieved $\mathrm{N}^{2}$ scaling. Information derived from QM is used to develop a force field, which is an empirical functional form, for atomistic-level simulations for use in Molecular Dynamics (MD) techniques. Though MD simulations can be used to study system sizes $\sim 10^{5}$ larger than QM, but at times material design requires the need to simulate larger time-length scales $(\mu \mathrm{s} / \mathrm{s}-\mu \mathrm{m} / \mathrm{mm})$ than MD can handle. This leads to the next steps, considering grains (Meso-scale) and grids (Finite elements) for design and evaluation of materials.

The objective of the molecular modeling investigations at the Jet Propulsion Laboratory (JPL) was to provide theoretical support for organic and inorganic sensor materials evaluation, design, and selection for the JPL Electronic Nose (ENose) [5-9]. The JPL ENose is an array-based sensing instrument designed and built for near realtime air quality monitoring in crew habitat aboard the space shuttle/space station. The JPL ENose is designed to run continuously and to monitor for the presence of selected chemical species in the air at parts-per-million (ppm) to parts-per-billion (ppb) concentration ranges. The Third Generation JPL ENose operated onboard the International Space Station (ISS) as a Technology Demonstration for seven months during 2008-2009 (Fig. 16.1). In the previous two generations, the target analyte list consisted of more than 20 organic solvents, ammonia, hydrazine, and freon113 [9]. The chemical species targeted for the Technology Demonstration included a subset of previously targeted analytes as well as three additional new species: elemental mercury $(\mathrm{Hg})$, sulfur dioxide $\left(\mathrm{SO}_{2}\right)$, and Freon 218.

The previous two JPL ENose generations each consisted of an array of 32 sensing films made from polymer-carbon composites. In order to detect the entire suite of organic and inorganic analytes for the Third Generation JPL ENose, a combination of organic and inorganic sensing materials were investigated.

Polymer (organic) based sensing films were the preferred choice for sensing materials in the sensor development efforts for the Third Generation JPL ENose. This is not only due to the ease of integration of new polymer-based sensors into the existing 


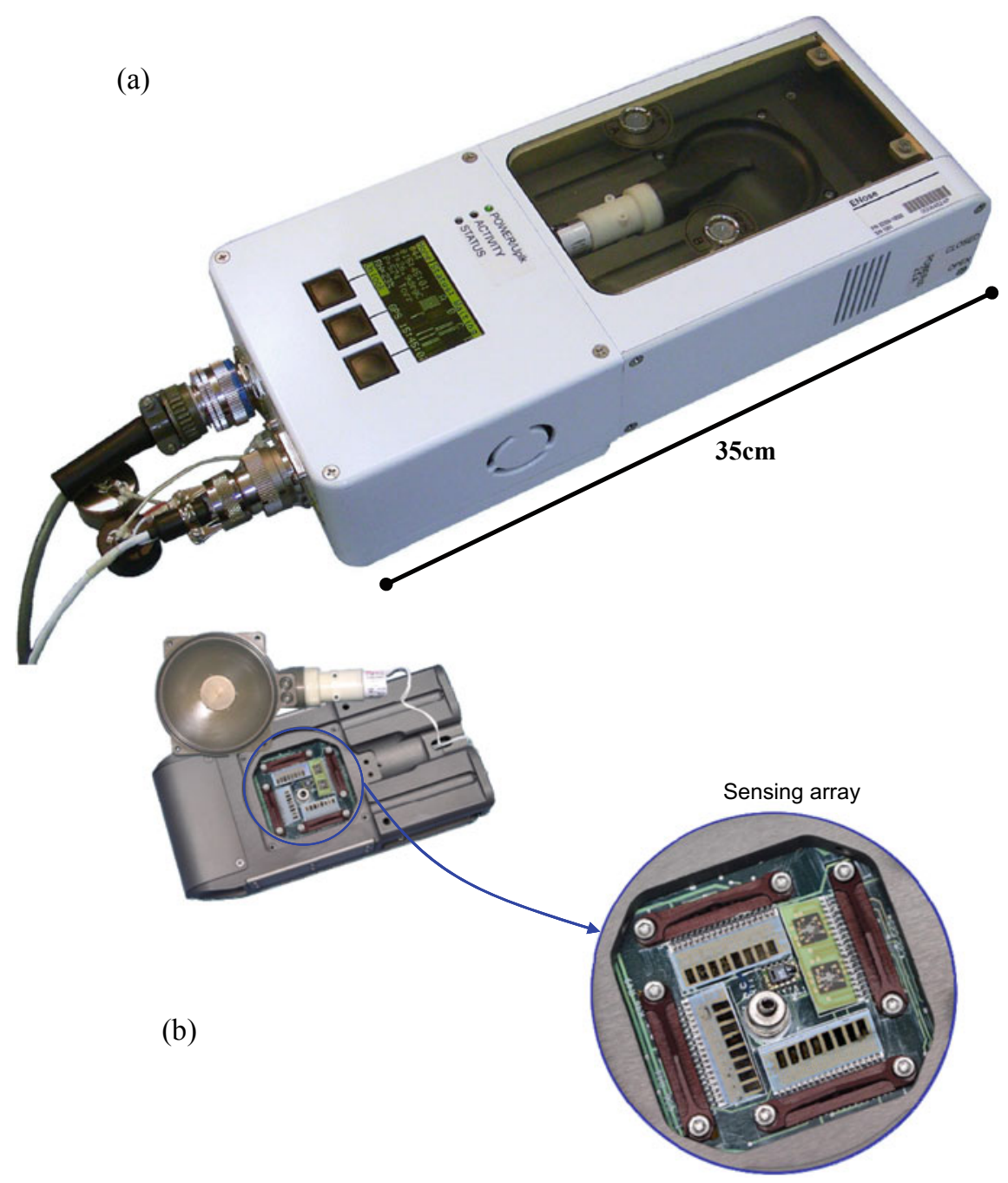

Fig. 16.1 a The Third-Generation JPL ENose for the Technology Demonstration onboard ISS. Sensor unit is enclosed in the Interface Unit which was connected to the ISS EXPRESS Rack, b sensing Chamber of the JPL ENose sensor unit. Seen are sensor array on 4 chips $(8$ sensors per chip) optimized for target analytes

JPL ENose platform, but also because the polymeric sensors can be easily operated/regenerated at temperature conditions close to or slightly elevated above room temperatures. Moreover, polymer-based sensing can be tailored to provide a wide range of chemical and physical properties. Hence for modeling screening, organic groups were chosen as the potential sensing material candidates, as they could be a part of a polymer chain, either in the backbone or as side groups. These organic 
groups include alkanes, alkenes, aromatics, primary and secondary amines, amides, and carboxylic acids.

This chapter will focus on a first principles QM approach to screen organic chemical functionalities to detect vapors of $\mathrm{SO}_{2}$ and elemental $\mathrm{Hg}$ by calculating the binding energies between the organics and the target molecules. To validate the QM findings, materials containing recommended chemical functionalities were then tested and the experimental result trends compared with the binding energy trends.

\subsection{Understanding and Selection of Polymer-Carbon Sensing Arrays}

The conductivity of the polymer-carbon composite films in the JPL ENose is due to the conductive pathways formed by the carbon black particles in the polymer matrix. The mechanism of sensor response in polymer-carbon composite sensing films is, to first order, based on the swelling of the sensing films. Swelling of the sensing films on exposure to target molecules occurs due to the sorption of the target molecules as a result of partitioning. This is a direct result of the interactions of the target molecules with the polymer matrix. The swelling disrupts conductive pathways of the carbon particles in polymer matrix, resulting in an increase in electronic resistance in the film $[8,10-12]$. This change in resistance is measured and is used to construct the pattern of response across the sensing array (the "fingerprint"). Other mechanisms that may also contribute to a change in resistance in polymer-carbon composite sensors by interrupting conductive pathways are ionization of analyte in water trapped in the film or analyte sorption to carbon particles. Hence, to detect a target analyte, an understanding of the binding (or interaction) energies between the polymer and the target molecules is important to provide us a guideline for chemical functionalities in polymers to be used in the sensing materials screening. We have developed molecular models to understand these mechanisms and the chemical and physical characteristics that govern them [13].

Selection of polymeric sensing materials for an ENose array is based on achieving distinctive patterns across the array, for all the target analytes of interest. The JPL ENose sensor array selection has been a three-step process [8]. First, responses of polymers to a subset of target analytes are measured to provide data for further analysis. Second, a statistical analysis of measured responses of polymers to representative analytes (selected by functional group) is used to determine the best distribution of patterns of response across the analytes. The selection of the sensor set and their corresponding weights are optimized by maximizing distances between gas signatures. Finally, a set of polymers is selected from the polymers indicated by statistical analysis as providing a high degree of definition from one analyte to the next. In the final selection, polymer films which show the lowest-noise response and a high degree of reproducibility of response are chosen. The polymers used in 
the first generation ENose were selected based only on statistical analysis; chemical functionality was not considered.

The polymers selected for the second generation represent five categories [9] of chemical functionality:

Hydrogen-Bond Acidic (HBA), Hydrogen-Bond Basic (HBB), Dipolar and Hydrogen-Bond Basic (D-HBB), Moderately Dipolar, and weakly H-Bond basic or acidic (MD-HB), and Weakly Dipolar with weak or no hydrogen-bond properties (WD). Based on these categories, polymers were selected for experimental testing and statistical analyses were performed on the data. A final set of sensors were selected through this process.

The objective of this investigation is to provide theoretical support for organic sensor materials selection for $\mathrm{SO}_{2}$ and $\mathrm{Hg}$ detection. This chapter will discuss the computational evaluation of sensing materials using first principles quantum mechanics approach and experimental results to validate the theoretical findings. The screening methodology involves calculating binding energies for organic sensor materials with the $\mathrm{SO}_{2}$ and $\mathrm{Hg}$ molecules.

\subsection{Rapid Screening Using Quantum Mechanics}

The quantum mechanics tools are used for rapid screening of common classes of organic groups which may be considered to be a part of a polymer chain, either in the backbone or as side groups, to detect $\mathrm{SO}_{2}$ and $\mathrm{Hg}$. The system sizes considered are only a few tens of atoms, and hence first principles techniques will be used to provide an accurate binding energy value for organics- $\mathrm{SO}_{2}$ and organics-Hg systems. The organic functionalities include alkanes (ethane), alkenes (ethylene), aromatics (benzene), primary, secondary amines (monomethyl, and dimethyl amines), amide (formamide), and carboxylic groups (formic acid). We have also screened organics that have a combination of chemical functionalities, as in the case of methylpyrollidone, that contains a carbonyl group, along with nitrogen in an aromatic ring and connected to a methyl (alkane) group. The organics considered for this work are shown in Fig. 16.2.

Density Functional Theory (DFT) B3LYP and in selected cases with a newly developed flavor of DFT, X3LYP method was used to perform ground electronic state energy calculations. To validate the DFT method's accuracy for elemental $\mathrm{Hg}$ systems, we calculated the first and second ionization potentials for gold $(\mathrm{Au})$ and elemental $\mathrm{Hg}$ atoms in the gas phase. The basis set of Ross et al. [14] was employed for cases containing $\mathrm{Hg}$ and LACVP**++ basis set for $\mathrm{Au}$. The predicted and experimental values match well [15].

Jaguar quantum mechanics program [16] with the pseudo-spectral grid method $[17,18]$ was used for calculating the electronic energy of each molecule (organics, $\mathrm{SO}_{2}$ and $\mathrm{Hg}$ ) in vacuum at $0{ }^{\circ} \mathrm{K}$. The gas-phase geometries of all complexes were optimized until the energy converged to better than $5.0 \times 10^{-5}$ Hartrees (1 Hartrees 
<smiles>CC</smiles>

(a)<smiles>CN</smiles>

(d)<smiles>O=CO</smiles>

(g)<smiles>C=C</smiles>

(b)<smiles>CNC</smiles>

(e)<smiles>CN1CCCC1=O</smiles>

(h)<smiles>c1ccccc1</smiles>

(c)<smiles>NC=O</smiles>

(f)

Fig. 16.2 Organics used for quantum mechanics rapid screening to detect $\mathrm{SO}_{2}$ and $\mathrm{Hg}$. The chemical functional groups are listed in the parenthesis after the organic molecule. a Ethane (alkane), b ethylene (alkenes), c benzene (aromatics), d, e monomethyl and dimethyl amines (primary, secondary amines), $\mathbf{f}$ formamide (amide), $\mathbf{g}$ formic acid (carboxylic groups) and $\mathbf{h}$ methylpyrollidone (carbonyl, amine group in a ring)

$=627.50955 \mathrm{kcal} / \mathrm{mol}$ ), using a fine pseudo-spectral grid. The procedure previously discussed [15] employs the steps to:

(i) Optimize molecular geometries of the organic compounds in gas phase.

(ii) Create the organic- $\mathrm{SO}_{2}$, or [the organic- $\mathrm{Hg}$ ] complexes by positioning the sulfur or $\mathrm{Hg}$ atom away from the electron-rich positions $(\mathrm{X})$ in each organic molecule. Various original starting orientations were employed, in some cases, such as for ethane, ethylene, and benzene. For example in the ethane-Hg system, axial approach and perpendicular approach of the mercury atom to the C-C axis were considered.

(iii) Perform energy minimization for the organics- $\mathrm{SO}_{2}, \mathrm{Hg}$ complexes, to obtain the starting geometry to conduct a distance scan of the potential energy surface. Single point energy calculations were performed every $0.1 \AA$ from 2.5 to $6.0 \AA$, as the scan distance $\mathrm{R}\left(\mathrm{SO}_{2}, \mathrm{Hg}, \ldots, \mathrm{X}\right)$ was varied. During these calculations, the "binding" angle and dihedral angles from this minimized structure were left as obtained by the full geometry minimization of the complexes. Counterpoise (basis set superposition error) corrections were performed for $r=6.0 \AA$ only, and the value of this correction applied to all other distances. This procedure results in errors no larger than $0.1 \mathrm{kcal} / \mathrm{mol}$. The binding energy for the organics$\mathrm{SO}_{2}$ and organics-Hg systems are defined as 


$$
\begin{gathered}
E_{\text {bind }_{\text {organic-SO }}}=E_{\mathrm{DFT}}\left(\text { organic }-\mathrm{SO}_{2}\right)-\left[E_{\mathrm{DFT}}\left(\mathrm{SO}_{2}\right)+E_{\mathrm{DFT}}(\text { organic })\right] \\
E_{\text {bind }_{\text {organic-Hg }}}=E_{\mathrm{DFT}}(\text { organic }-\mathrm{Hg})-\left[E_{\mathrm{DFT}}(\mathrm{Hg})+E_{\mathrm{DFT}}(\text { organic })\right],
\end{gathered}
$$

where $E_{\mathrm{DFT}}$ is the calculated DFT energy. The counterpoise basis set superposition error (BSSE) corrections are performed for $R=6.0 \AA$ only, and the value of this correction applied to all other distances in calculating the binding energy $[17,18]$. The binding energies of organics- $-\mathrm{SO}_{2}$ and $\mathrm{Hg}$ complexes calculated at varying scan distances were also, used to develop force field parameters for molecular dynamics (MD) simulations.

\subsection{Results and Discussion}

\subsubsection{Screening and Validation of Organics for $\mathrm{SO}_{2}$ Detection}

The binding energy $\left(\mathrm{E}_{\text {bind }}\right)$ results for the organics- $\mathrm{SO}_{2}$ systems are reported in Table 16.1. The chemical functionalities that show strong $\mathrm{SO}_{2}$ binding energies $(<-10 \mathrm{kcal} / \mathrm{mol})$ are amine, amide, and acid compounds. Weak and or no binding of $\mathrm{SO}_{2}$ observed with alkane, alkene, and aromatic hydrocarbon compounds. The binding energies follow the series (most favorable to least favorable): Amines $>$ Amides $>$ Aldehydes $>$ Acids $>$ Aromatics $>$ Alkenes $>$ Alkanes.

We have also analyzed the van der Waals and electrostatic energy term contributions in the binding energy, for the interactions between the organics and $\mathrm{SO}_{2}$ molecules. The energy contributions due to the electrostatic interactions are greater than the van der Waals interactions for the amines, carboxylic acid, aldehyde, and amide groups. On an atomic level analysis of binding energy for the dimethylamine$\mathrm{SO}_{2}$ system (highest binding energy), it was found that the sulfur atom of $\mathrm{SO}_{2}$

Table 16.1 Quantum Mechanics binding energies for organics- $\mathrm{SO}_{2}$ systems

\begin{tabular}{l|l}
\hline System & Binding energy kcal/mol \\
\hline Dimethylamine- $\mathrm{SO}_{2}$ & -12.54 \\
\hline Methylamine- $\mathrm{SO}_{2}$ & -10.93 \\
\hline Formamide- $\mathrm{SO}_{2}$ & -9.86 \\
\hline Formic acid- $\mathrm{SO}_{2}$ & -6.43 \\
\hline $\mathrm{n}-$ Methylpyrrolidone- $\mathrm{SO}_{2}$ & -4.64 \\
\hline Benzene- $\mathrm{SO}_{2}$ & -2.42 \\
\hline Ethylene- $\mathrm{SO}_{2}$ & -2.2 \\
\hline Ethane- $\mathrm{SO}_{2}$ & -0.57 \\
\hline
\end{tabular}


has maximum contributions to the total van der Waals interaction energy, with sulfur-nitrogen pair being the primary contributor.

Based on the above recommendations, we designed and synthesized new polymers EYN2 and EYN7 to detect $\mathrm{SO}_{2}$ at 1 ppm concentration levels. These are poly-4-vinyl pyridine derivatives with a quaternary and a primary amine. The chemical structures of the polymers are shown in Fig. 16.3. The two polymers were made into polymercarbon black composite sensors using protocols that have been previously described [19]. These films were loaded with $8-10 \%$ carbon by weight and solution deposited onto microhotplate sensors substrates with a sensor area of $4 \times 10^{-8} \mathrm{~m}^{2}(200 \mu \mathrm{m}$ by $200 \mu \mathrm{m})$. Sensors were exposed to $0.2-20 \mathrm{ppm}$ of $\mathrm{SO}_{2}$ in air at $22-25^{\circ} \mathrm{C}$ and relative humidity of $\sim 30 \%\left(\sim 10,000 \mathrm{ppm}_{2} \mathrm{O}\right)$. As predicted by the QM binding energies

EYN1

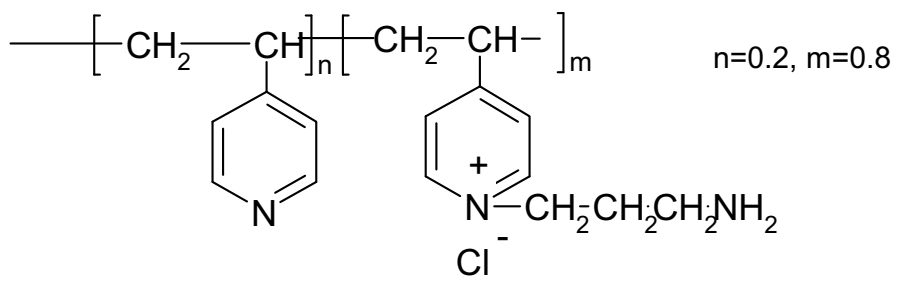

EYN2<smiles>CC(C)CC(CC(C)(C)C)c1cc[n+](CCN)cc1</smiles><smiles>CCCCCCc1cc[n+](-c2ccc(CN)cc2)cc1</smiles>

EYN7

Fig. 16.3 Polymers synthesized for detection of $\mathrm{SO}_{2}$ and elemental $\mathrm{Hg}$ vapors 


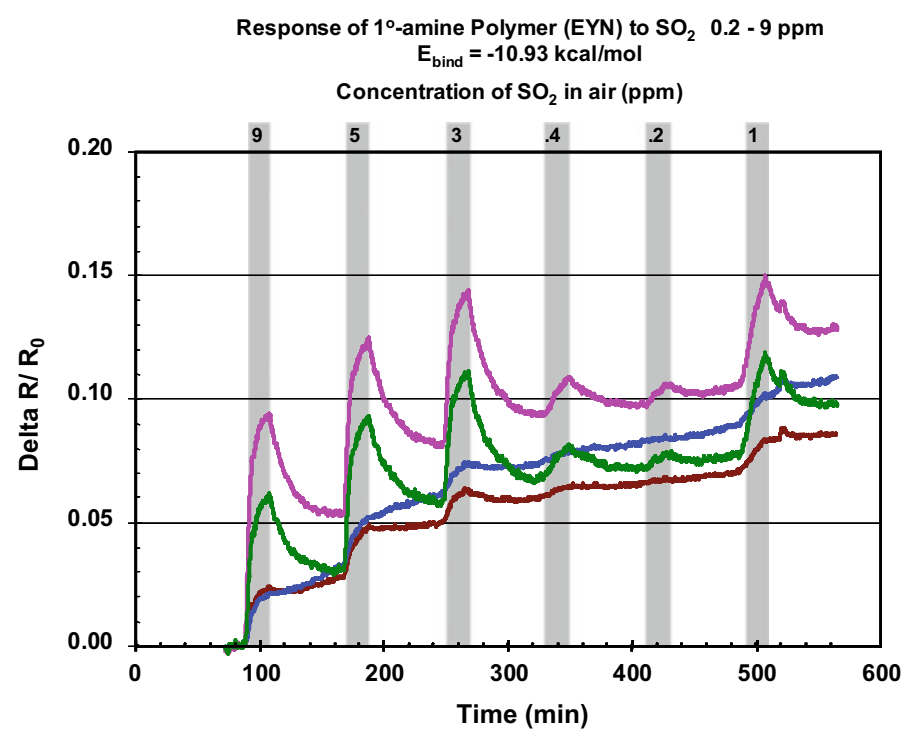

Fig. 16.4 Sensor response of EYN-carbon composites to $\mathrm{SO}_{2}$ at room temperature $\left(22-25^{\circ} \mathrm{C}\right)$ and $30 \%$ relative humidity

between $\mathrm{SO}_{2}$ and amines, Fig. 16.4 shows a strong response of two micro-sensors made from polymer EYN2 to concentrations from 0.2 to $9 \mathrm{ppm} \mathrm{SO}_{2}$. The baseline rise in the figure is caused by drift in environmental temperature during the test.

We also tested other polymer-carbon composites with different polymer chemical functionalities, used in previous JPL ENose generations. As can be seen in Fig. 16.5, consistent with the QM binding energy trends, some polymers responded to $10-15 \mathrm{ppm} \mathrm{SO}_{2}$ in air and some did not. The two polymers which responded relatively strongly to the presence of $\mathrm{SO}_{2}$ are polyimide and polyamide; both functionalities are predicted to respond to $\mathrm{SO}_{2}$. The polycaprolactone polymer responded moderately well to $\mathrm{SO}_{2}$. The polycaprolactone polymer contains an ester functionality (carboxylic acid derivative), which may be expected to respond to $\mathrm{SO}_{2}$ if the acid shows such behavior. Also responding very weakly (but measurably) to $\mathrm{SO}_{2}$ is polyvinylacetate (\#6), which contains an ester functionality. Weak or no sensor response is seen for polymers \#4 and \#5. Polymer \#4 contains ethane and ethene functionalities, and polymer $\# 5$ has an aromatic functionality; in both cases, the responses are consistent with the weak binding energies of the corresponding functional group.

It is interesting to note that the responses for the previously selected polymer composite films are one order of magnitude smaller for one order of magnitude larger $\mathrm{SO}_{2}$ concentration, than responses for the amines selected from the modeling work. This response size is also consistent with the predicted interaction energies for these functionalities; primary and secondary amines were predicted to have the largest interaction energy (absolute value) and ethane was predicted to have the smallest (absolute value). 


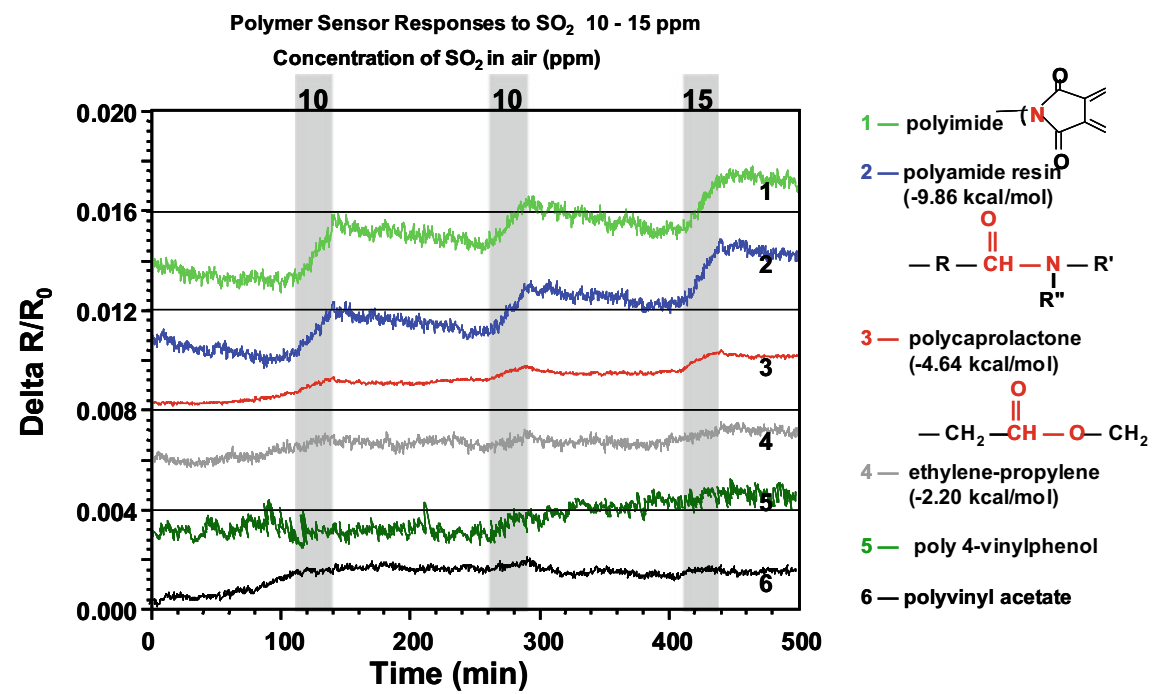

Fig. 16.5 Sensor responses on previous JPL ENose polymer-carbon composites to $\mathrm{SO}_{2}$ at room temperature $\left(22-25^{\circ} \mathrm{C}\right)$ and $30 \%$ relative humidity. Shown are the functional group and the binding energy values

\subsubsection{Screening and Validation of Organics for Elemental Hg Detection}

The binding energy results obtained using quantum mechanics for organic- $\mathrm{Hg}$ systems are tabulated in Table 16.2. It can be seen that a polymer candidate for elemental $\mathrm{Hg}$ vapor detection would be one containing amine functional groups, preferably primary or secondary. The amine functionalities interact weakly with elemental $\mathrm{Hg}$, with binding energy values $<-0.4 \mathrm{kcal} / \mathrm{mole}$. This would lead us to expect that a polymer-carbon composite sensor made from a polymer containing

Table 16.2 Quantum mechanics binding energies for organics-Hg systems

\begin{tabular}{l|l}
\hline System & Binding energy kcal/mol \\
\hline Dimethylamine-Hg & -0.33 \\
\hline Methylamine- $\mathrm{Hg}_{2}$ & -0.38 \\
\hline Formamide- $\mathrm{Hg}$ & $>0$ \\
\hline Formic acid-Hg & $>0$ \\
\hline n-Methylpyrrolidone-Hg & $>0$ \\
\hline Benzene- $\mathrm{Hg}$ & $>0$ \\
\hline Ethylene- $\mathrm{Hg}$ & $>0$ \\
\hline Ethane- $\mathrm{Hg}$ & $>0$
\end{tabular}

The binding energies greater than zero indicate no binding 
primary or secondary amine functionalities, would show a weak response to the presence of vapor-phase mercury. No binding was shown for other functional groups modeled. The binding energies of organics- $\mathrm{Hg}$ systems only have contributions from the van der Waals energy term. This is because of the zero charges on the elemental mercury atom. As discussed in the previous section, the organics- $\mathrm{SO}_{2}$ systems, has both electrostatic and van der Waals contributions. The electrostatic contributions are from the interactions of the partial charges on the sulfur and oxygen atoms in the $\mathrm{SO}_{2}$ molecule and those on the atoms of the organic molecules.

Based on the QM binding energy results between mercury and organic ligands, two polymers were selected and made into polymer-carbon black composite sensors. These two polymers are EYN2 and EYN1. Like EYN2 (discussed previously), EYN1 also is a poly-4-vinyl pyridine derivative with a quaternary and a primary amine. These films were loaded with $10-15 \%$ carbon by weight and solution cast onto microhotplate sensor substrates and onto ENose substrates. It was expected from the small magnitude of the binding energy that any response of sensors made from these polymers would be weak.

As shown in Fig. 16.6, polymer EYN1, with a primary and quaternary amine in its structure, has a weak response to mercury vapor at concentrations of $10 \mathrm{ppb}$ and

\section{Response of Polymer (EYN) to $\mathrm{Hg} 9$ - $47 \mathrm{ppb}$ $E_{\text {bind }}=-0.38 \mathrm{kcal} / \mathrm{mol}$}

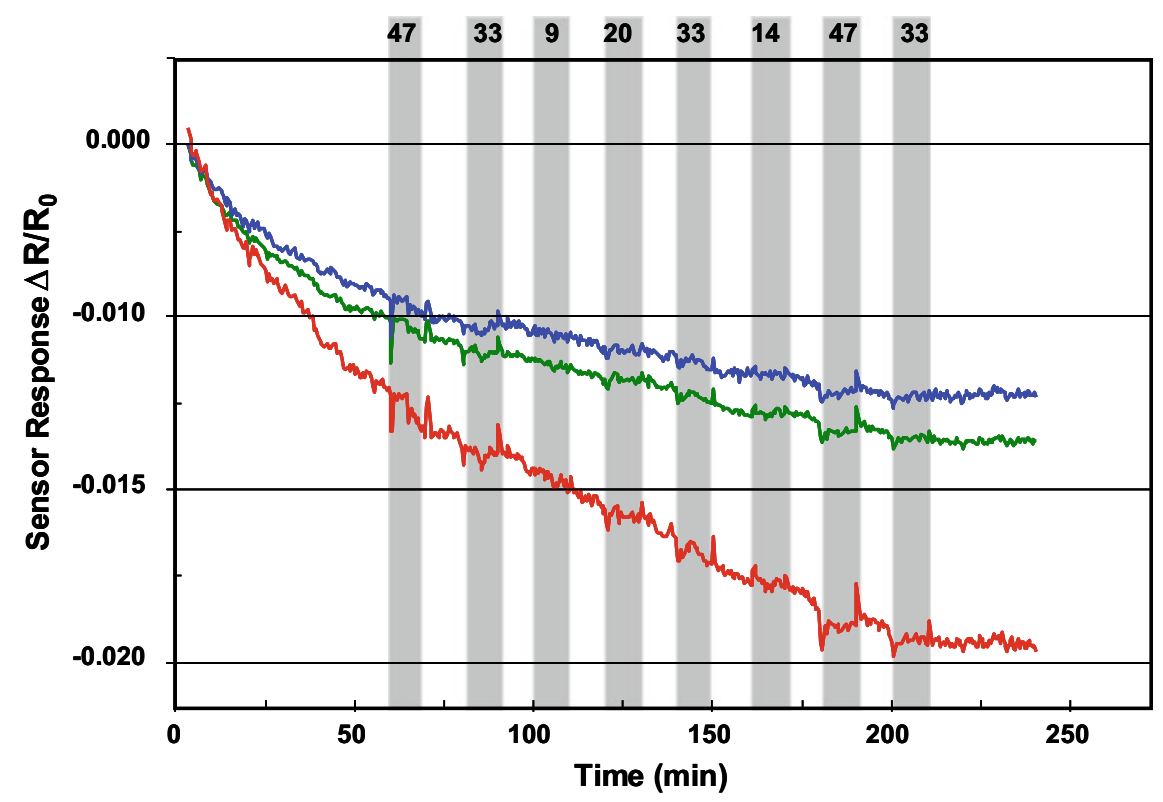

Fig. 16.6 Weak sensor response of EYN polymer-carbon composites to elemental $\mathrm{Hg}$ at room temperature $\left(22-25^{\circ} \mathrm{C}\right)$ and $30 \%$ relative humidity 
higher at room temperatures at $30 \%$ relative humidity. This level of response is not suitable for a mercury sensor at these concentrations; however, the response is validation of the results of the modeled interaction. Further work and optimization would be necessary to use these materials as mercury sensors at higher concentrations.

Polymers used in the first and second generation JPL ENose were also tested for response to $\mathrm{Hg}$ in humidified air. As expected based on modeled results, no polymers responded to the presence of $\mathrm{Hg}$ in the $\mathrm{ppb}$ range. Imide, amide, and amine-containing polymers showed a very weak response at $\mathrm{Hg}$ concentrations well above the required range.

A Morse force field generated by doing an empirical fit to the binding energy curves [15] will be used to evaluate the performance of other polymers to detect $\mathrm{SO}_{2}$ and $\mathrm{Hg}$. The force field will also be used to calculate the chemical interactions between the analytes and the selected polymer-based sensors. A sensor response model was developed [20] by applying Quantitative Structure Activity Relationships (QSAR) techniques using Genetic Function Approximations (GFA). The sensor response model correlates sensor activity and molecular descriptors. The model was used to predict array sensor responses of new and untested target analytes, as well as to predict chemical identity of molecules producing an "unknown" array response [21].

\subsection{Conclusions}

We have developed a rapid sensing materials screening approach using first principles, for detecting sulfur dioxide $\left(\mathrm{SO}_{2}\right)$ and elemental mercury $(\mathrm{Hg})$ vapors. The approach involves calculating binding energies of common organic functionalities suitable for the fabrication of polymer-based sensors. These organic groups include alkanes, alkenes, aromatics, primary and secondary amines, amides, and carboxylic acids. The calculated binding energies for organic- $\mathrm{SO}_{2}$ and organic-Hg systems indicate that a polymer candidate for both $\mathrm{SO}_{2}$ and $\mathrm{Hg}$ detection would be one containing primary or secondary amines. Other chemical functionalities in the polymer that have strong binding with $\mathrm{SO}_{2}$ are amides and acids. To validate the QM findings, materials containing recommended chemical functionalities have been tested and the experimental results compared with calculated results. Experimental results show that this approach is a good method for ranking the performance of various sensing materials to detect $\mathrm{SO}_{2}$ and $\mathrm{Hg}$ or in general any target analytes. The sensors were successfully used in the Third-Generation JPL ENose Technology Demonstration Experiment on ISS.

Acknowledgements The research reported in this chapter was carried out at the Jet Propulsion Laboratory, California Institute of Technology under a contract with the National Aeronautics and Space Administration and supported by the Advanced Environmental Monitoring and Control Program, ESMD, NASA. Copyright 2018 California Institute of Technology. Government sponsorship acknowledged. 


\section{References}

1. R.A. Potyrailo, Polymeric sensor materials: toward an alliance of combinatorial and rational design tools? Angew. Chem. Int. Ed. 45(5), 702-723 (2006)

2. T.J. Koplin, M. Siemons, C. Ocen-Valentin, D. Sanders, U. Simon, Workflow for high throughput screening of gas sensing materials. Sensors 6(4), 298-307 (2006)

3. M. Kubo, T. Kubota, C. Jung, K. Seki, S. Takami, N. Koizumi, K. Omata, M. Yamada, A. Miyamo, Combinatorial computational chemistry approach to the high-throughput screening of metal sulfide catalysts for CO hydrogenation process. Energy Fuels 17, 857-861 (2003)

4. W.A. Goddard, T. Cagin, M. Blanco, N. Vaidehi, S. Dasgupta, W. Floriano, M. Belmares, J. Kua, G. Zamanakos, S. Kashihara, M. Iotov, G.H. Gao, Strategies for multiscale modeling and simulation of organic materials: polymers and biopolymers. Comput. Theor. Polym. Sci. 11, 329-343 (2001)

5. M.A. Ryan, M.L. Homer, M.G. Buehler, K.S. Manatt, F. Zee, J. Graf, Monitoring the air quality in a closed chamber using an electronic nose, in Proceedings of 27th International Conference on Environmental Systems (ICES), Nevada, USA (1997)

6. M.A. Ryan, M.L. Homer, M.G. Buehler, K.S. Manatt, B. Lau, D. Karmon, S. Jackson, Monitoring space shuttle air for selected contaminants using an electronic nose, in Proceedings of 28th International Conference on Environmental Systems (ICES), Massachusetts, USA (1998)

7. M.A. Ryan, M.L. Homer, H. Zhou, K.S. Manatt, V.S. Ryan, S. Jackson, Operation of an electronic nose aboard the space shuttle and directions for research for a second generation device, in Proceedings of 30th International Conference on Environmental Systems (ICES), Toulouse, France (2000)

8. M.A. Ryan, H. Zhou, M.G. Buehler, K.S. Manatt, V.S. Mowrey, S.P. Jackson, A.K. Kisor, A.V. Shevade, M.L. Homer, Monitoring space shuttle air quality using the JPL electronic nose. IEEE Sens. J. 4, 337-347 (2004)

9. M.A. Ryan, A.V. Shevade, H. Zhou, M.L. Homer, Polymer-carbon-composite sensors for an electronic nose air quality monitor. MRS Bull. 29, 714-719 (2004)

10. E.K. Sichel (ed.), Carbon Black-Polymer Composites (Marcel Dekker, New York, 1982)

11. M.C. Lonergan, E.J. Severin, B.J. Doleman, S.A. Beaber, R.H. Grubb, N.S. Lewis, Arraybased vapor sensing using chemically sensitive, carbon black-polymer resistors. Chem. Mater. 8, 2298-2312 (1996)

12. M.S. Freund, N.S. Lewis, A chemically diverse conducting polymer-based electronic nose. Proc. Natl. Acad. Sci. U. S. A. 92, 2652-2656 (1995)

13. A.V. Shevade, M.A. Ryan, M.L. Homer, A.M. Manfreda, H. Zhou, K.S. Manatt, Molecular modeling of polymer composite-analyte interactions in electronic nose sensors. Sens. Actuators B Chem. 93(1-3), 84-91 (2003)

14. R.B. Ross, J.M. Powers, T. Atashroo, W.C. Ermler, L.A. Lajohn, P.A. Christiansen, J. Chem. Phys. 93, 6654 (1990)

15. M. Blanco, A.V. Shevade, M.A. Ryan, Quantum mechanics and first-principles molecular dynamics selection of polymer sensing materials, in Computational Methods for Sensor Material Selection, ed. by M.A. Ryan, A.V. Shevade, C.J. Taylor, M.L. Homer, M. Blanco, J.R. Stetter. Integrated Microanalytical Systems (Springer, New York, Chap. 3, 2009)

16. L. Schrodinger, Jaguar 5.5, Release 11 Portland, Oregon (2003)

17. D. Chasman, M.D. Beachy, L.M. Wang, R.A. Friesner, Parallel pseudospectral electronic structure: I. Hartree-Fock calculations. J. Comput. Chem. 19, 017-1029 (1998)

18. R.A. Friesner, J.A. Bentley, M. Menou, C. Leforestier, Adiabatic pseudospectral methods for multidimensional vibrational potentials. J. Chem. Phys. 99, 324-335 (1993)

19. M.A. Ryan, M.L. Homer, H. Zhou, K. Manatt, A. Manfreda, A. Kisor, A. Shevade, S.P.S. Yen, Expanding the capabilities of the JPL electronic nose for an international space station technology demonstration, in Proceedings of 36th International Conference on Environmental Systems (ICES), Norfolk, USA 
20. A.V. Shevade, M.L. Homer, C.J. Taylor, H. Zhou, A.D. Jewell, K.S. Manatt, A.K. Kisor, S.P.S. Yen, M.A. Ryan, Correlating polymer-carbon composite sensor response with molecular descriptors. J. Electrochem. Soc. 153, H209-H216 (2006)

21. A.V. Shevade, M.L. Homer, H. Zhou, K.S. Manatt, A.K. Kisor, S.-P.S. Yen, L.M. Lara, M.A. Ryan, The technology demonstration of the third generation JPL electronic nose on the international space station, in Human Olfactory Displays and Interfaces: Odor Sensing and Presentation, ed. by T. Nakomoto (2013) 